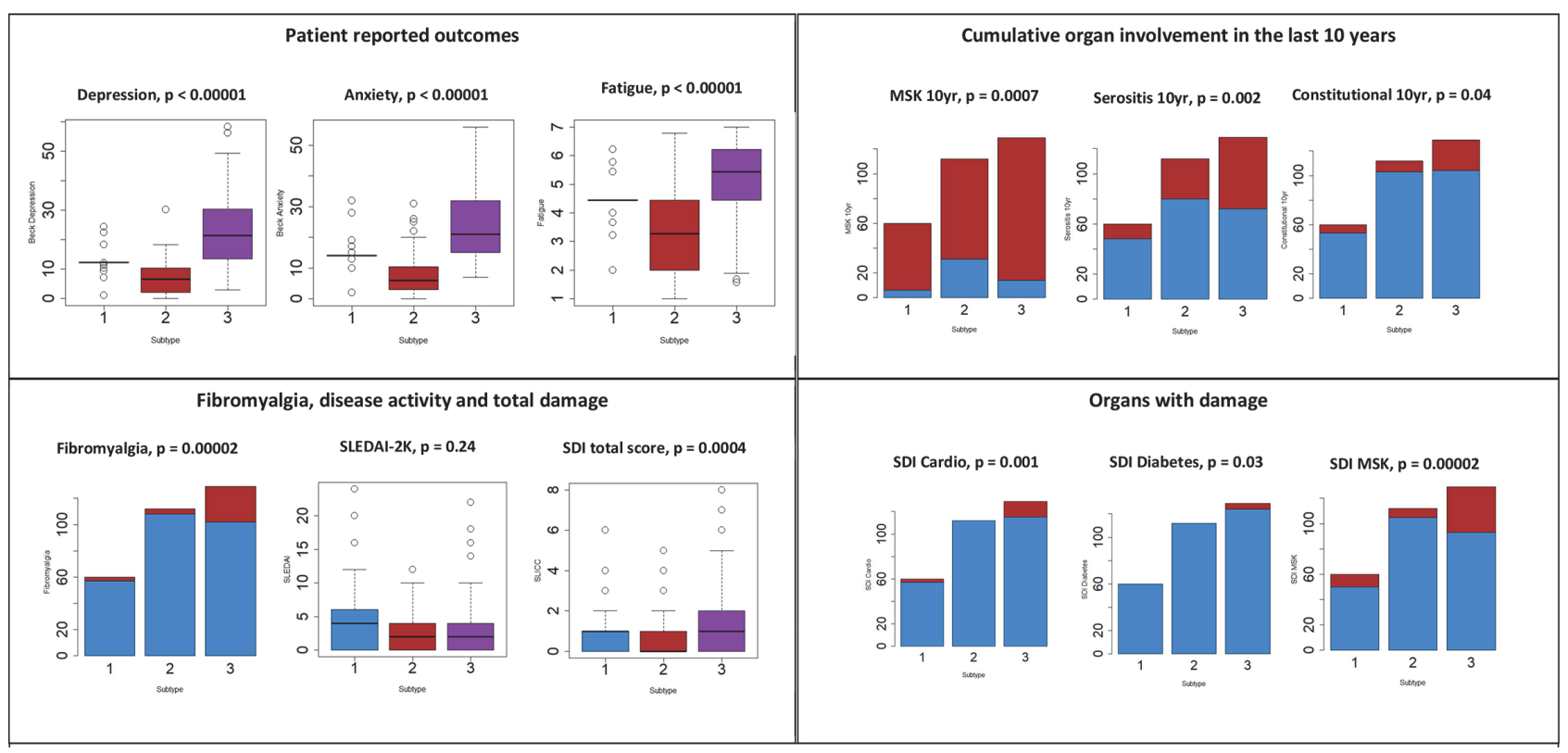

Abstract 1117 Figure 1 Variables with significant differences between the three phenotyped subtypes. Box and whisker plots: blue=subtype 1, red =subtype and purple=subtype 3. Bar charts: red=number of participants with variable and blue=number without

disease damage, Subtype 2 had lower levels and Subtype 1 mixed levels.

Conclusion The subtype with the greatest psychiatric and disease burden and reduced HRQoL performed worse on cognitive testing, specifically in domains of language processing and executive function. This subtype also had more musculoskeletal (MSK) and cardiovascular involvement. MSK involvement affects pain levels, which can impact cognition. Cardiovascular damage may be linked to cerebral small vessel disease, which is known to affect cognitive function SLE patients. Overall, these results aid with phenotyping CI in SLE and provide a baseline for our future longitudinal results.

\section{INCIDENCE OF SYSTEMIC LUPUS ERYTHEMATOSUS IN THE UNITED STATES: ESTIMATES FROM A META- ANALYSIS OF THE CENTERS FOR DISEASE CONTROL AND PREVENTION NATIONAL LUPUS REGISTRIES}

${ }^{1}$ Peter M Izmirly*, ${ }^{2}$ Elizabeth D Ferucci, ${ }^{3}$ Emily C Somers, ${ }^{3}$ Lu Wang, ${ }^{3}$ W Joseph McCune, ${ }^{4}$ S Sam Lim, ${ }^{4}$ Cristina Drenkard, ${ }^{5}$ Maria Dall'Era, ${ }^{6}$ Caroline Gordon, ${ }^{7}$ Charles G Helmick, ${ }^{8}$ Hilary Parton. ${ }^{1}$ New York University School of Medicine, USA; ${ }^{2}$ Alaska Native Tribal Health Consortium, Anchorage, USA; ${ }^{3}$ University of Michigan School of Medicine, USA; ${ }^{4}$ Emory School of Medicine, USA; ${ }^{5}$ University of California at San Francisco School of Medicine, USA; ${ }^{6}$ University of Birmingham, UK; ${ }^{7}$ Centers for Disease Control and Prevention, USA; ${ }^{8} \mathrm{New}$ York City Department of Health and Mental Hygiene, USA

\subsection{6/lupus-2021-lupus21century.61}

Background Epidemiologic data on systemic lupus erythematosus (SLE) are limited, particularly for racial/ethnic subpopulations in the United States (U.S.). This meta-analysis leveraged data from the Centers for Disease Control and Prevention (CDC) National Lupus Registry network of population-based SLE registries to estimate the general and by sex, race/ethnicity incidence of SLE in the U.S.

Methods The CDC registries were established in Michigan, Georgia, California, New York and through the Indian Health
Service (IHS). Registries used the 1997 revised ACR classification criteria for SLE as their case definition, and the surveillance time periods ranged from 2002-2009. Age-standardized incidence rates were stratified by sex and race/ethnicity from the state-based registries; the American Indian/Alaska Native (AI/AN) estimate was based only on the IHS registry that covered multiple states. For pooling data across the four sites with data on different racial/ethnic groups, we used Cochran's $\mathrm{Q}$ and $\mathrm{I}^{2}$ statistic to test for heterogeneity across sites. Due to significant heterogeneity, we used a random effects model to calculate pooled incidence, which allows for more variation across sites. We then extrapolated to the 2018 Census population data according to sex and race-stratified groups, including data from the IHS registry, and summed the stratum-specific estimates to provide a total population estimate of incident SLE cases in the U.S.

Results The registries contributed 1,057 classified cases of SLE from a mix of urban and rural areas. From the meta-analysis of the four state-based registries, the overall incidence was 5.1 (95\%CI4.6,5.6) per 100,000 person-years. The incidence among females was about 7 times higher than males (8.7 vs 1.2). In the meta-analysis, the incidence rate was highest among Black females $(15.9,95 \%$ CI12.5,20.3), followed by Asian/Pacific Islander females $(7.6,95 \%$ CI5.5,10.4), Hispanic females (6.8,95\%CI6.2,7.6), and White females $(5.7,95 \%$ CI4.9,6.7). Among males, the incidence rate was highest among Black males $(2.4,95 \% \mathrm{CI} 1.8,3.0)$ followed by Hispanic males $(0.9,95 \% \mathrm{CI} 0.4,1.9)$, White males $(0.8,95 \% \mathrm{CI} 0.6,1.1)$, and Asian/Pacific Islander males $(0.4,95 \% \mathrm{CI} 0.2,0.6)$. The AI/ AN incidence estimates, had the second highest rates of SLE among females $(10.4,95 \%$ CI6.6,14.6) and highest for males (3.8, 95\%CI1.6,7.8). Applying our sex- and race-specific incidence estimates to the corresponding population denominators from 2018 Census data, we estimated that 14,263 new persons (12,560 females and 1,703 males) in the U.S. were diagnosed with SLE and fulfill the ACR classification criteria, table 1. 
Abstract 1118 Table 1 Estimated Number of New Persons Diagnosed with Systemic Lupus Erythematosus in the United States in 2018

\begin{tabular}{|c|c|c|c|c|c|}
\hline & \multicolumn{5}{|c|}{ FEMALE } \\
\hline $\begin{array}{l}\text { Race/Ethnicity } \\
\text { (Number of sites in }\end{array}$ & \multicolumn{2}{|c|}{$\begin{array}{l}\text { SLE Incidence a }^{\text {a }} \\
\text { per 100,000 }(95 \% \text { Cl) }\end{array}$} & $\begin{array}{l}\text { Population } \\
\text { Denominator }\end{array}$ & \multicolumn{2}{|c|}{$\begin{array}{l}\text { Estimated \# SLE Cases } \\
\text { in United States }(95 \% \mathrm{Cl}\end{array}$} \\
\hline \multicolumn{6}{|l|}{ Race } \\
\hline Black (4) & 15.9 & $(12.5,20.3)$ & $24,880,722$ & 3,956 & $(3,110,5051)$ \\
\hline White (4) & 5.7 & $(4.9,6.7)$ & $130,137,989$ & 7,418 & $(6,377,8719)$ \\
\hline Asian/PI (2) & 7.6 & $(5.5,10.4)$ & $12,544,896$ & 953 & $(6,90,1305)$ \\
\hline Al/AN (1) & 10.4 & $(6.6,14.6)$ & $2,238,966$ & 233 & $(148,327)$ \\
\hline Total $^{\mathrm{b}}$ & 8.7 & $(8.1,9.4)$ & $169,802,573$ & 12,560 & $(10,325,15,402)$ \\
\hline \multicolumn{6}{|l|}{ Ethnicity } \\
\hline \multirow[t]{4}{*}{ Hispanic ${ }^{c}(2)$} & 6.8 & $(6.2,7.6)$ & $30,689,083$ & 2,087 & $(1,903,2332)$ \\
\hline & \multicolumn{2}{|c|}{ MALE } & & & \\
\hline & \multicolumn{2}{|c|}{ Incidence ${ }^{a}$} & Population & \multicolumn{2}{|c|}{ Estimated \# SLE Cases } \\
\hline & \multicolumn{2}{|c|}{ per $100,000(95 \% \mathrm{Cl})$} & Denominator & \multicolumn{2}{|c|}{ in United States $(95 \% \mathrm{Cl}$} \\
\hline \multicolumn{6}{|l|}{ Race } \\
\hline Black (4) & 2.4 & $(1.8,3.2)$ & $22,961,129$ & 551 & $(413,735)$ \\
\hline White (4) & 0.8 & $(0.6,1.1)$ & $127,942,583$ & 1,024 & $(768,1,407)$ \\
\hline Asian/PI (2) & 0.4 & $(0.2,0.6)$ & $11,660,533$ & 47 & $(23,70)$ \\
\hline Al/AN (1) & 3.8 & $(1.6,7.8)$ & $2,134,870$ & 81 & $(34,167)$ \\
\hline Total $^{b}$ & 1.2 & $(0.9,1.6)$ & $164,699,115$ & 1,703 & $(1,238,2,379)$ \\
\hline \multicolumn{6}{|l|}{ Ethnicity } \\
\hline Hispanic ${ }^{c}(2)$ & 0.9 & $(0.4,1.9)$ & $31,281,605$ & 282 & $(125,594)$ \\
\hline
\end{tabular}

Systemic lupus erythematosus cases were defined according to the 1997 revised American College of Rheumatology criteria.

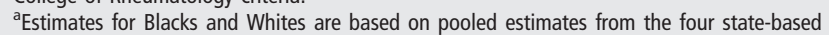
registries; Asian/Pacific Islanders (Asian/PI) and Hispanics are based on pooled estimates from California and New York; American Indian/Alaska Native (Al/AN) estimates are based on the Indian Health Service Registry.


(Asian/PI). Since the American Indian/Alaska Native (Al/AN) incidence was based on one registry and was significantly higher, it was not included in the pooled incidence per 100,000 .

'Hispanic ethnicity is not mutually exclusive from the race categories, i.e., all Hispanic persons are included in one of the race categories. Thus, the pooled estimates do not incorporate the Hispanic rates since that would lead to duplicate counting. Estimates for Hispanics are based on pooled estimates from California and New York.

Conclusion A coordinated network of population-based SLE registries provided more accurate estimates of the incidence of SLE and the numbers of new individuals affected with SLE in the U.S. in 2018.

\section{RECRUITMENT AND ENROLLMENT INTO A DIRECT-TO- FAMILY PEDIATRIC LUPUS TRIAL}

Rachel L Randell, Lindsay Singler, Anthony Cunningham, Laura E Schanberg*, Michael Cohen-Wolkowiez, Christoph P Hornik, Stephen J Balevic. Duke Clinical Research Institute, Duke University School of Medicine, Durham, NC, USA

\subsection{6/lupus-2021-lupus21century.62}

Background A direct-to-family or virtual approach can improve participant recruitment, retention, and diversity, and improve efficiency of clinical trials. Pediatrics and rare disease may especially benefit from this approach. Recruitment and enrollment of direct-to-family trials in pediatric lupus has not been described. We hypothesize that engaging patients and leveraging a disease registry will facilitate recruitment and enrollment into a direct-to-family pediatric lupus trial (NCT: 04358302).

Objective Evaluate the feasibility of enrolling and recruiting participants into a nationwide, novel direct-to-family pediatric lupus trial

Methods Investigators and study leaders collaborated with patients and advocacy groups, key stakeholders, and a disease registry (Childhood Arthritis and Rheumatology Research Alliance, CARRA Registry) to design a direct-to-family pediatric lupus trial. Participants across the country were identified through the CARRA Registry. Recruitment and informed consent/assent were conducted remotely by a single site (Duke Clinical Research Institute, DCRI).

Results 191 potentially eligible participants were identified through the CARRA Registry. Of the 84 participants who participated in a live discussion, 44 (52\%) scheduled a consenting call. The original enrollment goal of 20 participants was met in 10 days. Enrollment was increased to 26, and an additional 18 participants were added to a back-up list due to high interest.

Conclusions Using a single site to recruit and enroll participants into a nationwide direct-to-family pediatric lupus trial was highly feasible, quick, and effective. Recruiting participants from a disease registry and engaging patient advocacy groups were key to successful enrollment.

\section{DETERMINATION OF THE MINIMAL CLINICALLY IMPORTANT DIFFERENCE (MCID) OF THE PHYSICIAN GLOBAL ASSESSMENT (PGA) IN SLE}

Erik Anderson, Meggan Mackay, Cynthia Aranow*. Feinstein Institutes for Medical Research, Manhasset, USA

\subsection{6/lupus-2021-lupus21century.63}

Introduction The PGA is a SLE outcome measurement reflecting the physician's assessment of a patient's disease activity. It is considered a 'gold standard' and contributes to responder indices for clinical trials, as well as to definitions of the low lupus disease activity state (LLDAS) and remission. Although a few studies have evaluated its sensitivity to change, no studies have assessed its minimal clinically important difference (MCID). Our objective was to determine the MCID of the PGA using prospective data collected in a single center.

Methods PGAs were scored prospectively by 3 physicians on consecutive patient visits; the difference between a visit PGA and the previous visit's PGA was the $\triangle$ PGA. A disease flare visit was defined as a visit in which therapy was increased (initiation or increase of corticosteroid dose and/or DMARD or biologic agent). We constructed a receiver operating characteristics (ROC) curve to visualize the performance of the $\triangle$ PGA for predicting flare and determined the MCID of the PGA for flare using an anchored approach by calculating Youden's index for the $\triangle \mathrm{PGA}$ in 0.1 increments.

Results Data from 129 patient visits with $\triangle \mathrm{PGA}$ and therapeutic decisions (therapy increase/flare or therapy decreased or no change/no flare) were available. The baseline PGA was between $0-0.9$ in 79 visits, 1.0-1.9 in 38 visits and between 2.0-3 in 12 visits. Flare occurred in 85 visits while no flare occurred in 44 visits with $43(50.6 \%)$ of flares occurring with a baseline PGA between 0-0.9, 30 (35.3\%) with a baseline PGA between 1-1.9 and 12 (14.1\%) flares with a baseline 